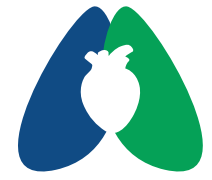

ASSOBRAFIR

C I Ê N C I A

\title{
Interrupção do sono na unidade de terapia intensiva: revisão integrativa acerca dos fatores associados
}

\author{
Sleep disruption in the intensive care unit: an integrative review of \\ associated factors
}

Anthony Medeiros Calado de Lima1*; Brena Patricia Silva do Carmo'; Carlos José Oliveira de Matos ${ }^{1}$

\begin{abstract}
Resumo
Introdução: O sono é um estado comportamental caracterizado pela diminuição das respostas aos estímulos do ambiente externo. É regulado pelo sistema nervoso central por meio de mecanismos fisiológicos e é dividido em sono Non Rapid Eye Movement (NREM) e sono Rapid Eye Movement (REM). Possui função reparadora ao mesmo tempo que promove o descanso. Na unidade de terapia intensiva (UTI), ambiente complexo e de monitorização constante, as atividades técnicas são sobrepostas à garantia do sono de qualidade dos pacientes não sedados, causando a interrupção deste processo. Método: trata-se de uma revisão integrativa, na qual a busca ocorreu por meio das bases de dados: PUBMED, LILACS (Literatura-Americana e do Caribe em Ciências da Saúde), Scielo (Scientific Electronic Library Online), Scopus, utilizando os descritores: intensive care units, sleep e patients; entre os anos de 2013 e 2018. Objetivo: Estudo com o objetivo de descrever os fatores que estão associados a interrupção do sono em pacientes não sedados na unidade de terapia intensiva. Resultados: Dentre os elementos que interrompem o sono foram identificados como os principais: a iluminação, grande quantidade de ruídos no ambiente de cuidados intensivos e manipulações excessivas durante a noite. Outros aspectos observados englobam a percepção dos profissionais sobre a importância desse processo fisiológico, porém não acompanhada de ações que garantam a sua qualidade, tal qual o impacto na função cerebral associada a sua interrupção. Conclusão: Foi possível constatar que os fatores ambientais da unidade de terapia intensiva (UTI) interferem diretamente na continuidade do sono, bem como a escassez de protocolos resulta em tomadas de decisões inadequadas quanto à preservação do tempo de descanso do paciente. Ruídos, iluminação excessiva e padrões emocionais como a ansiedade e o medo são as maiores causas de interrupção do sono. A ausência de protocolos para a promoção do sono na UTI implica na falta de ações adequadas executadas pelos profissionais. A ocorrência de delirium devido a diminuição da função cognitiva, o declínio do sistema imune e o comprometimento dos músculos respiratórios dificultando o desmame da ventilação mecânica, foram identificados como os desfechos associados a interrupção do sono de pacientes críticos.
\end{abstract}

Palavras-chave: Unidades de Terapia Intensiva; Sono; Privação do Sono; Cuidados Críticos; Assistência ao Paciente; Qualidade da Assistência à Saúde.

'Universidade Federal de Sergipe (UFS), Lagarto, SE, Brasil

Como citar: Lima AMC, Carmo BPS, Matos CJO. Interrupção do sono na unidade de terapia intensiva: revisão integrativa acerca dos fatores associados. ASSOBRAFIR Ciênc. 2020;11:e76523. https://doi. org/10.47066/2177-9333.AC.2020.0006

Submissão em: Setembro 28, 2019 Aceito em: Setembro 25, 2020

Estudo realizado em: Universidade Federal de Sergipe, Lagarto, SE, Brasil. Aprovação ética: Não se aplica

*Autor correspondente:

Anthony Medeiros Calado de Lima. E-mail: anthonymedeiroscaladodelima@gmail.com

\section{Abstract}

Background: Sleep is a behavioural state characterized by decrease in responses to stimuli in the external environment. Is regulated by the central nervous system through physiological mechanisms and is divided into Non Rapid Eye Movement sleep (NREM) sleep and Rapid Eye Movement (REM). Has restorative function to the time it promotes relaxation. In the intensive care unit (ICU), complex environment and constant monitoring, technical activities are superimposed to the guarantee of quality of sleep patients sedated, causing the interruption of this process. Aim: Study aiming to describe the factors that are associated with the interruption of sleep in patients non sedated in the intensive care unit. Method: this is an integrative review, in which the search occurred through the databases: PUBMED, LILACS (American and Caribbean Literature in Health Sciences), Scielo (Scientific Electronic Library Online), Scopus, using the descriptors: intensive care units, sleep and patients, between years 2013 and 2018. Results: Among the elements that disrupt their sleep were identified as key: lighting, lot of noises in intensive care environment and excessive manipulations 
during the night. Other aspects observed include the perception of professionals about the importance of this physiological process, but not accompanied by actions that ensure your quality, such as the impact on brain function associated with your interruption. Conclusion: It was found that the environmental factors of the intensive care unit (ICU) interfere directly on sleep continuity, as well as the scarcity of protocols results in inadequate decision-making regarding the preservation of the rest of the time patient. Noises, excessive lighting and emotional patterns as the anxiety and fear are the major causes of interruption of sleep. The absence of protocols for promoting sleep in ICU implies the lack of appropriate actions performed by professionals. The occurrence of delirium due to decreased cognitive function, the decline of the immune system and the commitment of respiratory muscles complicating weaning from mechanical ventilation, were identified as the outcomes associated with the interruption of sleep critical patients.

Keywords: Intensive Care Unit; Sleep; Sleep Deprivation; Critical Care; Patient Care; Quality of Healthcare.

\section{INTRODUÇÃO}

O sono é uma condição ativa, em que o cérebro e o corpo estão ativos durante esse estado comportamental, o qual é caracterizado por um aumento do limiar de excitação e diminuição das respostas aos estímulos externos, tal qual pela perda da consciência e pela postura variável, que, comumente, ocorre em decúbito. O sono é regulado homeostaticamente e pelo sistema circadiano, que modela o tempo e a propensão a dormir de acordo com mecanismos internos e externos ${ }^{1}$.

O sono é um estado parcial de desligamento reversível do ambiente e envolve mecanismos fisiológicos de grande complexidade junto ao sistema nervoso central. Tem função essencial para o descanso, reparação e sobrevivência, propiciando a recuperação do corpo depois de um dia cansativo e agindo de forma reparadora do corpo e da mente, sendo a sua privação uma das causas conhecidas pela diminuição da resistência à infecções ${ }^{2,3}$. Além disso, promove a eliminação de substâncias possivelmente neurotóxicas do sistema nervoso central (SNC) e, dessa maneira, sustenta a homeostase metabólica4.

No sono normal há padrões característicos de ativação, que podem ser verificados por eletroencefalografia (EEG), e que estão associadas as suas fases: NREM, controlado por mecanismos homeostáticos, é subdividido nos estágios 1 (que refere-se a transição entre o sono e o despertar), 2 (ocorrência dos chamados fusos do sono) e 3 (predominância de baixa frequência e alta amplitude no EEG). Além disso, o sono Non Rapid Eye Movement sleep (NREM) possui fundamental importância nos processos restauradores, como na restauração da memória. A fase Rapid Eye Movement sleep (REM) é caracterizada pelos movimentos oculares rápidos e controlado pelo ritmo circadiano, representa cerca de $20 \%$ do tempo total de sono. É uma fase reparadora do sono e onde acontecem os sonhos ${ }^{1,5}$.

O ciclo circadiano pode ser definido como um ciclo de 24 horas sobre o qual é baseado o ciclo biológico do ser humano. É regulado pelo núcleo supraquiasmático do hipotálamo e ajuda a determinar o ciclo sono-vigília, tende a ser de 24 horas pois tem grande influência dos estímulos do ambiente, com maior predominância da luminosidade e é interrompido facilmente em ambientes isentos de turno claro/escuro ${ }^{3}$. O sono adequado é essencial para a manutenção da saúde, porém uma parcela significativa da população adulta sofre com alterações no ciclo sonovigília, ou seja, no período em que se está acordado e no período em que se está dormindo 4 .
No âmbito hospitalar, a UTI é um ambiente de alta complexidade, com monitorização e observação constante. Com isso, os aspectos físicos e técnico do paciente são colocados como prioridade em detrimento aos aspectos humanizados do cuidado ${ }^{2}$. Nesse local pacientes tem uma má qualidade do sono, em que vários fatores podem contribuir para o declínio do seu padrão. Os ruídos ambientais e a iluminação são um desses fatores, podendo ser descritos como o fator mais impactante na perturbação do sono tais como: conversas dos profissionais, sons emitidos pelos monitores, telefones e televisão ${ }^{2,3}$.

Assim, a privação do sono de pacientes críticos não sedados é uma grande preocupação, pois, juntamente com a incapacidade de dormir durante a hospitalização, é o segundo maior estressor e uma das três principais causas de ansiedade ao longo desse período de internação na $\mathrm{UTI}^{6}$. Há ainda a contribuição destes para outros desfechos negativos, como a ocorrência de delirium, suscetibilidade a infecções nosocomiais repetidas e aumento do período de internação, bem como elevação da mortalidade dos indivíduos em estado crítico ${ }^{6}$.

O interesse para o desenvolvimento deste artigo deu-se a partir da relação entre o sono e o desfecho clínico do paciente crítico, os quais são fatores pouco analisados e discutidos nesse ambiente. O presente estudo tem como objetivo descrever os fatores que estão associados a interrupção do sono em pacientes não sedados na unidade de terapia intensiva.

\section{METODOLOGIA}

O presente estudo trata-se de uma revisão integrativa, elaborada a partir das seguintes etapas: (1) elaboração da pergunta norteadora; (2) busca ou amostragem na literatura; (3) coleta de dados; (4) análise crítica dos estudos incluídos; (5) discussão dos resultados; (6) apresentação da revisão integrativa?.

Para nortear a revisão integrativa, formulou-se o seguinte questionamento: como as pesquisas têm abordado a qualidade do sono dos pacientes não sedados na unidade de terapia intensiva?

A busca ocorreu por meio das bases de dados: PUBMED, LILACS (Literatura-Americana e do Caribe em Ciências da Saúde), Scielo (Scientific Electronic Library Online), Scopus, utilizando os descritores: intensive care units, sleep e patients; e o operador boleano AND. Para fim de refinamento da pesquisa durante a busca nas bases de 
dados já citadas, foram usados os filtros: texto completo disponível e ano de publicação entre 2013 e 2018.

Como critério de inclusão foi proposto: Artigos na íntegra escritos em inglês ou português, artigos publicados nos últimos 5 anos, e em consonância com os objetivos desta pesquisa. Foram excluídos, artigos de revisão, teses, dissertações, monografias, trabalhos de conclusão de curso, resenhas críticas e editoriais.

Após a seleção dos artigos, estes foram analisados esclarecendo: autor, ano de publicação, objetivos e conclusão. Para a análise e interpretação dos dados foi utilizado o método de análise de conteúdo. A apresentação e discussão dos dados foram feitas de maneira descritiva, a partir do método de categorização temática.

\section{RESULTADOS}

Foram incluídos 10 artigos após a seleção por meio dos critérios de inclusão. Estes foram analisados com base nos autores, periódico, ano de publicação, título, objetivos e conclusão.

Em relação ao ano de publicação dos estudos, foram encontradas 2 (20\%) referências em 2018, 3 (30\%) em 2017, 1 (10\%) em 2016, 3 (30\%) em 2015, nenhuma em 2014 e 1 (10\%) em 2013. A Figura 1 descreve o processo de seleção e inclusão dos artigos.

A partir da análise da essência do conteúdo, foi possível observar 6 (60\%) estudos, os quais evidenciam que os ruídos e a iluminação são uma das maiores causas de piora no sono dos pacientes na UTI, 3 (30\%) estudos mostram que a quantidade de manipulações é inversamente proporcional a um sono de qualidade, 1 (10\%) relata que não apenas os fatores ambientais influenciam no sono, mas também a emoção e o sentimento de ansiedade estão ligados à qualidade do sono, e 1 (10\%) traz que a falta de diretrizes para os profissionais resulta numa promoção inadequada do sono de qualidade. A Tabela 1 exibe informações sobre os estudos envolvidos.

\section{DISCUSSÃO}

A discussão foi dividida em 3 categorias de acordo com a similaridade do conteúdo. A primeira categoria referese aos fatores ambientais e emocionais que influenciam na qualidade e continuidade do sono, elencando os principais fatores que podem contribuir para um sono de má qualidade. A segunda categoria traz fatos relacionados a incapacitação dos profissionais acerca do assunto sono na UTI, mostrando o posicionamento dos profissionais e a importância de protocolos que os oriente a promover o sono adequado. A terceira categoria trata dos impactos da má qualidade do sono na saúde dos pacientes críticos, evidenciando as complicações e como estas podem alterar o desfecho do paciente.

Categoria 1 - Fatores ambientais e emocionais que influenciam na qualidade e continuidade do sono:

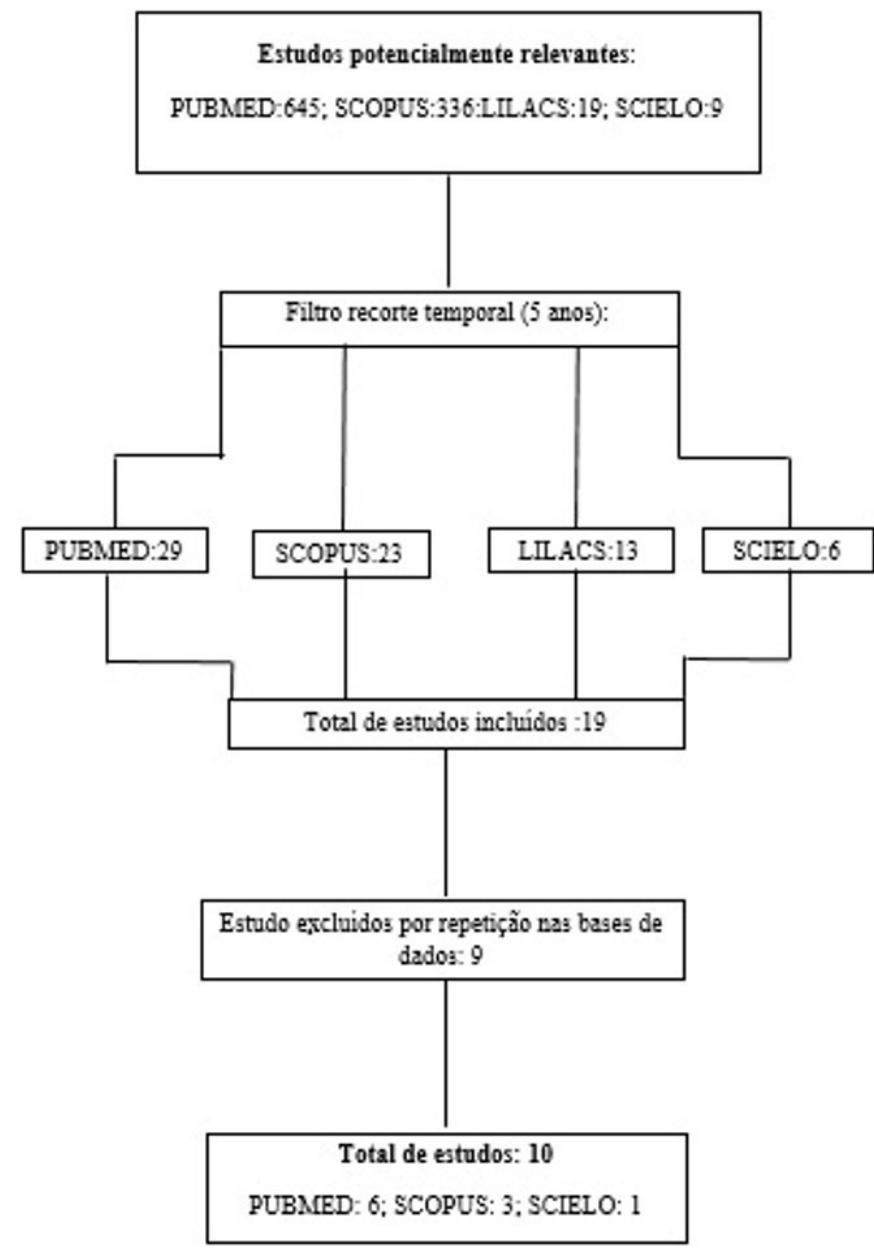

Figura 1. Critérios de inclusão e exclusão.

O ambiente da UTI é ruidoso, isso se comprova pela percepção de $100 \%$ dos membros de uma equipe clínica, que identificou o ruído como principal estressor ambiental ${ }^{12}$. Os ruídos são apontados como o fator mais preocupante na hora do sono ${ }^{9}$. Os sons mais perturbadores são: alarmes dos equipamentos e conversas da equipe clínica ${ }^{9}$. Podendo ser observado que em quartos individuais a intensidade de ruídos foram menores, porém todas as medições na UTI ultrapassaram os limites recomendados de intensidade sonora $(35 \mathrm{dBA})^{10,11}$. Em relação aos turnos, é possível observar que os ruídos durante o dia são mais elevados do que durante a noite ${ }^{10}$. Quanto ao sexo, mulheres relataram uma melhor qualidade do sono quando comparado aos do sexo masculino?.

A ansiedade é um sentimento relatado pela maioria dos pacientes, podendo ser causado pela falta do sono ou como resultado da dor. Além disso, o medo da morte na UTI contribui para o sentimento de ansiedade e para a privação do sono ${ }^{16}$.

Categoria 2 - Incapacitação dos profissionais acerca da promoção do sono de qualidade.

O fluxo imprevisível de trabalho causa perturbações ambientais durante a noite na unidade de terapia intensiva e é responsável por alterar o padrão de sono dos pacientes. Procedimentos de rotina estão associados 
Tabela 1. Caracterização dos estudos incluídos.

\begin{tabular}{|c|c|c|c|c|}
\hline Autores & Objetivos & $\begin{array}{c}\text { Método de } \\
\text { Análise }\end{array}$ & Resultados & Conclusão \\
\hline Pisani et al. ${ }^{8}$ & $\begin{array}{l}\text { Revisar métodos } \\
\text { para medir o sono } \\
\text { na UTI, incluindo } \\
\text { polissonografia, } \\
\text { actigrafia e } \\
\text { questionários. }\end{array}$ & $\begin{array}{l}\text { Análise de } \\
\text { conteúdo }\end{array}$ & $\begin{array}{l}\text { Os principais fatores para a privação } \\
\text { do sono dos pacientes na UTI são } \\
\text { a gravidade do doença subjacente, } \\
\text { dor, estresse e ansiedade } \\
\text { relacionada ao quadro clínico } \\
\text { e ao ambiente da UTI (ruídos e } \\
\text { iluminação noturna). }\end{array}$ & $\begin{array}{l}\text { O sono inadequado contribui } \\
\text { para a ocorrência de disfunção } \\
\text { cerebral na UTI. } \\
\text { A abordagem multidisciplinar } \\
\text { para a compreensão e } \\
\text { tratamento do problema } \\
\text { deve ser realizada de maneira } \\
\text { compromissada para que haja } \\
\text { melhora nos cuidados na UTI e } \\
\text { nos resultados do paciente. }\end{array}$ \\
\hline Simons et al. ${ }^{9}$ & $\begin{array}{l}\text { Determinar o efeito } \\
\text { do ruído na qualidade } \\
\text { subjetiva do sono. }\end{array}$ & $\begin{array}{l}\text { Análise de } \\
\text { conteúdo }\end{array}$ & $\begin{array}{l}\text { Entre os ruídos mais perturbadores, } \\
\text { os pacientes apontaram alarmes } \\
\text { dos equipamentos, fala e outras } \\
\text { atividades da equipe de saúde como } \\
\text { os mais constantes. }\end{array}$ & $\begin{array}{l}\text { Os níveis de ruído estão } \\
\text { associados negativamente aos } \\
\text { períodos de recuperação } \\
\text { Menores níveis de ruídos estão } \\
\text { associados positivamente à } \\
\text { qualidade subjetiva do sono em } \\
\text { pacientes de UTI. }\end{array}$ \\
\hline Elbaz et al. ${ }^{10}$ & $\begin{array}{l}\text { Avaliar o impacto dos } \\
\text { níveis de intensidade } \\
\text { sonora na UTI e suas } \\
\text { fontes no sono de } \\
\text { pacientes em UTI em } \\
\text { um período de } 24 \\
\text { horas }\end{array}$ & $\begin{array}{l}\text { Análise de } \\
\text { conteúdo }\end{array}$ & $\begin{array}{l}\text { Os ruídos mais prevalentes foram os } \\
\text { alarmes do monitor e do ventilador } \\
\text { mecânico. }\end{array}$ & $\begin{array}{l}\text { Na UTI a intensidade do nível do } \\
\text { som é um fator de perturbação } \\
\text { da continuidade do sono e deve } \\
\text { ser controlada num perfil de } \\
24 \text { horas. }\end{array}$ \\
\hline Tegnestedt et al. ${ }^{11}$ & $\begin{array}{l}\text { Investigar se o } \\
\text { tamanho da sala afeta } \\
\text { os níveis sonoros ou } \\
\text { a frequência de sons } \\
\text { perturbadores em } \\
\text { pacientes da unidade } \\
\text { de terapia intensiva. }\end{array}$ & $\begin{array}{l}\text { Análise de } \\
\text { conteúdo }\end{array}$ & $\begin{array}{l}\text { Os níveis sonoros mais elevados } \\
\text { foram identificados pela manhã } \\
\text { e pela tarde. Dentre os sons mais } \\
\text { perturbadores houve destaque, } \\
\text { respectivamente, para alarmes } \\
\text { de equipamentos e conversas } \\
\text { não relacionadas à assistência ao } \\
\text { paciente. }\end{array}$ & $\begin{array}{l}\text { Quartos com apenas uma } \\
\text { cama não garantem níveis de } \\
\text { som mais baixos, mas podem } \\
\text { implicar sons disruptivos menos } \\
\text { frequentes. Sessenta e quatro } \\
\text { por cento dos sons disruptivos } \\
\text { eram evitáveis. }\end{array}$ \\
\hline Ding et al. ${ }^{12}$ & $\begin{array}{l}\text { Explorar as } \\
\text { percepções e crenças } \\
\text { dos funcionários, } \\
\text { pacientes e } \\
\text { substitutos em } \\
\text { relação aos fatores } \\
\text { ambientais e não } \\
\text { ambientais na } \\
\text { unidade de terapia } \\
\text { intensiva que afetam } \\
\text { o sono dos pacientes. }\end{array}$ & $\begin{array}{l}\text { Análise de } \\
\text { conteúdo }\end{array}$ & $\begin{array}{l}\text { O principal fator ambiental } \\
\text { identificado foi o ruído. Interrupções } \\
\text { do sono eram mais frequentes } \\
\text { à noite e as causas relacionadas } \\
\text { consistiam em cuidados de rotina e } \\
\text { cuidados urgentes. } \\
\text { Fatores psicológicos afetam mais } \\
\text { intensamente o sono que os fatores } \\
\text { ambientais. }\end{array}$ & $\begin{array}{l}\text { As diminuições nas fontes } \\
\text { ambientais de perturbação } \\
\text { são necessárias, mas não são } \\
\text { suficientes para a melhora do } \\
\text { sono. } \\
\text { Emoções e ansiedade dos } \\
\text { pacientes devem ser abordas } \\
\text { e diretrizes devem orientar os } \\
\text { cuidados para proporcionar o } \\
\text { sono adequado. }\end{array}$ \\
\hline Kandar et al. ${ }^{13}$ & $\begin{array}{l}\text { Avaliar as percepções } \\
\text { e práticas dos } \\
\text { profissionais de } \\
\text { saúde em relação ao } \\
\text { sono na UTI. }\end{array}$ & $\begin{array}{l}\text { Análise de } \\
\text { conteúdo }\end{array}$ & $\begin{array}{l}\text { 75\% dos entrevistados indicaram } \\
\text { que os pacientes da UTI tiveram } \\
\text { sono "ruim" ou "muito ruim" sono } \\
\text { ruim; } 88 \% \text { afirmaram que o sono } \\
\text { de má qualidade pode afetar o } \\
\text { processo de recuperação na UTI. } \\
\text { Os entrevistados também sentiram } \\
\text { que o sono ruim estava associado a } \\
\text { resultados negativos na UTI, como o } \\
\text { desenvolvimento de delirium (97\%), } \\
\text { maior tempo de internação (88\%), } \\
\text { pouca participação na fisioterapia } \\
\text { (87\%) e liberação retardada da } \\
\text { ventilação mecânica (83\%). }\end{array}$ & $\begin{array}{l}\text { Embora a maioria dos } \\
\text { profissionais acredite que o } \\
\text { sono na UTI é ruim e implica } \\
\text { de maneira negativa nos } \\
\text { resultados do paciente, não há } \\
\text { protocolos de promoção do } \\
\text { sono nessas unidades. }\end{array}$ \\
\hline
\end{tabular}


Tabela 1. Continuação...

\begin{tabular}{|c|c|c|c|c|}
\hline Autores & Objetivos & $\begin{array}{l}\text { Método de } \\
\text { Análise }\end{array}$ & Resultados & Conclusão \\
\hline $\begin{array}{l}\text { Ritmala- } \\
\text { Castren et al. }{ }^{14}\end{array}$ & $\begin{array}{l}\text { Descrever a qualidade } \\
\text { do sono de pacientes } \\
\text { não intubados } \\
\text { e as atividades } \\
\text { de cuidados de } \\
\text { enfermagem } \\
\text { noturnos em uma } \\
\text { unidade de terapia } \\
\text { intensiva. }\end{array}$ & $\begin{array}{l}\text { Análise de } \\
\text { conteúdo }\end{array}$ & $\begin{array}{l}\text { Um maior número de atividades de } \\
\text { enfermagem foi significantemente } \\
\text { associado à redução do sono leve } \\
\text { e profundo. } 62 \% \text { dos cuidados } \\
\text { de enfermagem foram realizados } \\
\text { enquanto os pacientes estavam } \\
\text { acordados. }\end{array}$ & $\begin{array}{l}\text { Pacientes não intubados sofrem } \\
\text { de um sono tão inadequado } \\
\text { quanto os pacientes } \\
\text { submetidos à ventilação } \\
\text { mecânica na UTI } \\
\text { O planejamento mais cuidadoso } \\
\text { da assistência pode permitir } \\
\text { que os pacientes durmam mais } \\
90 \text { minutos de sono. }\end{array}$ \\
\hline \multirow[t]{2}{*}{ Naik et al. ${ }^{15}$} & $\begin{array}{l}\text { Avaliar a quantidade } \\
\text { e a qualidade do } \\
\text { sono em pacientes } \\
\text { internados na UTI, } \\
\text { usando actigrafia } \\
\text { e Questionário de } \\
\text { Sono de Richards- } \\
\text { Campbell (RCSQ), } \\
\text { respectivamente. }\end{array}$ & $\begin{array}{l}\text { Análise de } \\
\text { conteúdo }\end{array}$ & $\begin{array}{l}\text { Sono insatisfatório foi encontrado } \\
\text { em } 47 \% \text { dos pacientes. A prevalência } \\
\text { de sono insuficiente foi maior } \\
\text { entre os pacientes em ventilação } \\
\text { mecânica. Apenas 55,63\% do tempo } \\
\text { total de sono foi durante a noite. }\end{array}$ & $\begin{array}{l}\text { Há uma alta prevalência de } \\
\text { sono de má qualidade entre os } \\
\text { pacientes admitidos na UTI }\end{array}$ \\
\hline & $\begin{array}{l}\text { Conhecer a } \\
\text { perspectiva do } \\
\text { paciente sobre os } \\
\text { fatores relacionados } \\
\text { ao mau sono. }\end{array}$ & & & $\begin{array}{l}\text { É necessário minimizar as } \\
\text { intervenções prematuras } \\
\text { e projetar técnicas não } \\
\text { farmacológicas para permitir } \\
\text { que os pacientes durmam } \\
\text { confortavelmente. }\end{array}$ \\
\hline Ehlers et al. ${ }^{16}$ & $\begin{array}{l}\text { Identificar e descrever } \\
\text { os fatores que } \\
\text { contribuem para a } \\
\text { privação do sono } \\
\text { em uma unidade } \\
\text { multidisciplinar de } \\
\text { terapia intensiva } \\
\text { (UTIMU) em um } \\
\text { hospital privado na } \\
\text { África do Sul. }\end{array}$ & $\begin{array}{l}\text { Análise de } \\
\text { conteúdo }\end{array}$ & $\begin{array}{l}\text { Dos pacientes entrevistados, } 91,2 \% \\
\text { identificaram a dor como processo } \\
\text { contribuinte para a privação do } \\
\text { sono, } 88,2 \% \text { associaram o sono } \\
\text { de má qualidade a dispositivos } \\
\text { que limitavam seus movimentos; } \\
\text { 82,4\% afirmaram que os alarmes } \\
\text { e atividades em andamento } \\
\text { atrapalhavam o seu sono. }\end{array}$ & $\begin{array}{l}\text { Situações que deixam os } \\
\text { pacientes inseguros e ansiosos } \\
\text { foram relatadas como causas } \\
\text { para a privação do sono } \\
\text { durante a internação na UTI. } \\
\text { Não saber os nomes dos } \\
\text { profissionais de saúde, ruídos } \\
\text { causados por alarmes e a } \\
\text { restrição de movimentos devido } \\
\text { à presença de dispositivos } \\
\text { tiveram impacto significativo } \\
\text { na qualidade do sono dos } \\
\text { pacientes. }\end{array}$ \\
\hline Hamze et al. ${ }^{17}$ & $\begin{array}{l}\text { Identificar } \\
\text { intervenções } \\
\text { assistenciais, } \\
\text { realizadas pela } \\
\text { equipe de saúde, } \\
\text { e a influência } \\
\text { das mesmas na } \\
\text { continuidade do } \\
\text { sono de pacientes } \\
\text { internados em } \\
\text { Unidade de Terapia } \\
\text { Intensiva. }\end{array}$ & $\begin{array}{l}\text { Análise de } \\
\text { conteúdo }\end{array}$ & $\begin{array}{l}\text { Das } 529 \text { intervenções assistenciais } \\
\text { realizadas, } 21 \text { (4,0\%) causaram } \\
\text { interrupção no sono dos pacientes. } \\
\text { Dos } 28 \text { diferentes tipos de } \\
\text { intervenções assistenciais realizadas, } \\
12(42,8 \%) \text { causaram despertares } \\
\text { no sono dos pacientes. Foram } \\
\text { registrados, no total, } 21 \text { despertares. } \\
\text { Desses, } 13 \text { (62\%) ocorreram no } \\
\text { período diurno, entre } 7 \text { e } 13 \text { h, e oito } \\
\text { (38,0\%) no período noturno, entre } \\
\text { zero e } 3 \text { h. }\end{array}$ & $\begin{array}{l}\text { Intervenções assistenciais } \\
\text { podem prejudicar o sono dos } \\
\text { pacientes internados na UTI. }\end{array}$ \\
\hline
\end{tabular}

a essa interrupção, como mensuração dos sinais vitais, administração de medicamentos, avaliação do paciente, mensuração da glicemia capilar e alimentação oral12,17. Outros elementos citados como desconfortáveis nesse ambiente foram os cateteres de demora e endotraqueal ${ }^{15}$.

Segundo estudo desenvolvido sobre a percepção dos profissionais acerca do sono no ambiente de assistência intensiva, muitos o classifica como de má qualidade, estimam que seus pacientes dormem menos de 6 horas diárias e acreditam que esse período de hipovigília é importante, de modo que contribui para a recuperação do quadro clínico do indivíduo crítico ${ }^{13}$. Há, entretanto, divergência entre o pensamento e a prática clínica dos profissionais envolvidos no cuidado de pacientes graves, a partir da observação da consciência destes sobre a influência do sono na reabilitação do organismo, porém não acompanhada de práticas que promovam a ininterrupção desse processo ${ }^{13}$. Essa incongruência entre a percepção da importância do sono e os esforços para viabilizá-lo possivelmente reflete tanto a eficácia 
como o esforço necessário para que mudanças sejam alcançadas no cuidado (alteração dos fluxos de trabalho e agrupamento dos cuidados, por exemplo) e na ambiência da UTI por meio da regulação de ruídos e iluminação ${ }^{13}$.

As diretrizes atuais para melhorar a qualidade do sono de pacientes hospitalizados têm enfatizado a execução de ações multidisciplinares e integradas para agrupar o cuidado e reduzir a perturbação ambiental como meio de promoção do sono adequado na UTI ${ }^{12}$. Diante disso, pôde ser verificado que profissionais que dispunham de protocolos de promoção do sono em suas unidades de assistência intensiva puderam observar melhora da qualidade desse processo homeostático em seus pacientes em contrapartida àqueles que não tinham acesso a esses protocolos. Estes, associados às medidas de promoção que incluem agrupamento de atividades de assistência, regulação da luz e "horários de silêncio"13.

Os profissionais da UTI devem ter consciência do efeito da baixa qualidade do sono na promoção e reabilitação da saúde dos indivíduos aos quais prestam assistência ${ }^{14}$. Torna-se necessário que estes desenvolvam medidas que possibilitem a continuidade e qualidade do sono nesse ambiente normalmente estressor. Para isso a gestão hospitalar deve tornar a qualidade do sono dos pacientes numa preocupação constante e inerente à assistência prestada. A diminuição dos fluxos de trabalho noturno, quando possível, o agrupamento dos cuidados ao indivíduo criticamente doente, atenuando os episódios de interrupção do sono, bem como realizar adequações ambientais durante a noite para que o sono na UTI seja preservado são algumas alternativas.

Categoria 3 - Implicações da má qualidade do sono:

A privação do sono pode afetar, de maneira controversa, o sistema imunológico, a neurocognição, mecânica pulmonar e pode tornar os pacientes da UTI mais suscetíveis a infecções. Os hormônios da tireoide e os níveis de cortisol são diretamente afetados com o nível de sono. Os músculos respiratórios são acometidos de forma crítica, dificultando o desmame de pacientes mecanicamente ventilados na UTI, aumentando a morbidade e mortalidade dos pacientes internados nessas unidades. Apesar de não ser comprovado a relação entre a privação do sono e o delirium, os sintomas de pacientes privados de sono é semelhante ao estado delirante, prejudicando a memória, atenção, tempo de resposta ${ }^{8}$.

\section{CONCLUSÃO}

Os ruídos constituem a maior causa da interrupção do sono, tal qual a iluminação excessiva e padrões emocionais, como a ansiedade e o medo. Além disso, os procedimentos de rotina, quando mal alocados, contribuem para a descontinuidade desse estado de hipovigília.

\section{FONTE DE FINANCIAMENTO}

Não houve fonte de financiamento externo no presente estudo.

\section{CONFLITO DE INTERESSES}

Os autores desse artigo declaram que não houve conflito de interesse.

\section{REFERÊNCIAS}

1. Besedovsky L, Lange T, Haack M. The sleep-immune crosstalk in health and disease. Physiol Rev. 2019;99(3):1325-80. http:// dx.doi.org/10.1152/physrev.00010.2018. PMid:30920354.

2. Beltrami FG, Nguyen XL, Pichereau C, Maury E, Fleury B, Fagondes S. Sleep in the intensive care unit. J Bras Pneumol. 2015;41(6):539-46. http://dx.doi.org/10.1590/s180637562015000000056 . PMid:26785964.

3. Ritmala-Castren M, Virtanen I, Leivo S, Kaukonen KM, LeinoKilpi $\mathrm{H}$. Sleep and nursing care activities in an intensive care unit. Nurs Health Sci. 2015;17(3):354-61. http://dx.doi. org/10.1111/nhs.12195. PMid:25786544.

4. Ray S, Reddy A. Cross-talk between circadian clocks, sleep-wake cycles, and metabolic networks: dispelling the darkness. BioEssays. 2016;38(4):394-405. http://dx.doi. org/10.1002/bies.201500056. PMid:26866932.

5. Carskadon M, Dement W. Normal human sleep: an overview. In: Kryger MH, Roth T, Dement WC, editors. Principles and practice of sleep medicine. St. Louis: Elsevier Saunders; 2011. p. 16-26. http://dx.doi.org/10.1016/B978-1-4160-66453.00002-5.

6. Huang $H$, Jiang $L$, Shen L, Zhang G, Zhu B, Cheng J, et al. Impact of oral melatonin on critically ill adult patients with in ICU sleep deprivation: study protocol for a randomized controlled trial. Trials. 2014;15(1):327. http://dx.doi. org/10.1186/1745-6215-15-327. PMid:25135124.

7. Souza MT, Silva MD, Carvalho R. Revisão integrativa: o que é e como fazer. Einstein. 2010;8(1):102-6. http://dx.doi. org/10.1590/s1679-45082010rw1134. PMid:26761761.

8. Pisani MA, Friese RS, Gehlbach BK, Schwab RJ, Weinhouse $\mathrm{GL}$, Jones SF. Sleep in the intensive care unit. Am J Respir Crit Care Med. 2015;191(7):731-8. http://dx.doi.org/10.1164/ rccm.201411-2099CI. PMid:25594808.

9. Simons KS, Verweij E, Lemmens PMC, Jelfs S, Park M, Spronk $P E$, et al. Noise in instensive care unit and its influence on sleep quality: a multicenter observational study in dutch intensive care units. Crit Care. 2018;22(1):250-7. http:// dx.doi.org/10.1186/s13054-018-2182-y. PMid:30290829.

10. Elbaz M, Léger D, Sauvet F, Champigneulle B, Rio S, Strauss $M$, et al. Sound level intensity severely disrupts sleep in ventiladed ICU patients through a 24-h period: a preliminary 24-h of sleep stages and associated sound levels. Annals of Integrative Care. 2017;7(25):1-9.

11. Tegnestedt C, Gunther A, Reichard A, Bjurstrom R, Alvarsson $J$, Martling CR, et al. Levels and sources of sound in the intensive care unit: an observational study of three room types. Acta Anaesthesiol Scand. 2013;57(8):1041-50. http:// dx.doi.org/10.1111/aas.12138. PMid:23750596.

12. Ding Q, Redeker NS, Pisani MA, Yaggi HK, Knauert MP. Factores influencing patients sleep in the intensive care unit: perceptions of patients and clinical staff. Am J Crit Care. 2017;26(4):278-86. http://dx.doi.org/10.4037/ajcc2017333. PMid:28668912.

13. Kandar B, Knauert M, Jones S, Parsons E, Parthasarathy S, Pisani M. Perceptions and pratices regarding sleep in the 
intensive care unit: . a survey of 1,223 critical care providers. Ann Am Thorac Soc. 2016;13(8):1370-7. http://dx.doi. org/10.1513/AnnalsATS.201601-0870C. PMid:27104770.

14. Ritmala-Castren M, Virtanen I, Leivo S, Kaukonen K-M, Leino-Kilpi H. Sleep and nursing care actives in an intensive care unit. Nurs Health Sci. 2015;17(3):354-61. http://dx.doi. org/10.1111/nhs.12195. PMid:25786544.

15. Naik R, Gupta K, Soneja M, Elavarasi A, Sreenivas V, Sinha $S$. Sleep quality and quantity in intensive care unit patients: a cross-sectional study. Indian J Crit Care
Med. 2018;22(6):408-14. http://dx.doi.org/10.4103/ijccm. IJCCM_65_18. PMid:29962740.

16. Ehlers VJ, Watson $\mathrm{H}$, Moleki MM. Factors contributing the sleep deprivation in a multidisciplinary intensive care unit in South Africa. Curationis. 2013;36(1):1-8. http://dx.doi. org/10.4102/curationis.v36i1.72. PMid:23718136.

17. Hamze F, Souza C, Chianca T. Influência das intervenções assistenciais na continuidade do sono de pacientes na unidade de terapia intensiva. Rev Lat Am Enfermagem. 2015;23(5):789-96. http://dx.doi.org/10.1590/01041169.0514.2616. PMid:26487127. 